\title{
A case of posterior reversible encephalopathy syndrome in the setting of post-partum preeclampsia with suppressed plasma aldosterone levels and plasma renin activity
}

\author{
Aurelio Negro, Rosaria Santi, Chiara Grasselli, Simona Davoli, Franco Perazzoli \\ Second Division of Internal Medicine and Hypertension Unit, Arcispedale S. Maria Nuova, Reggio Emilia, Italy
}

\begin{abstract}
Posterior reversible encephalopathy syndrome (PRES) is characterized by headache, altered mental status, visual loss, and seizures. PRES is associated with neuroradiological findings: white matter abnormalities, predominantly in the parieto-occipital regions of the brain. PRES has been described in association with hypertensive encephalopathy, eclampsia, renal failure, or following immunosuppressive or anticancer therapy. We report a case of PRES in a severe preeclampsia occurring in the late postpartum period, with suppressed plasma aldosterone levels and plasma renin activity. These laboratory abnormalities may be due to an apparent mineralocorticoid excess syndrome.
\end{abstract}

\section{Introduction}

Posterior reversible encephalopathy syndrome (PRES) encompasses both clinical and radiological features, predominantly affecting the white matter of the posterior cerebral hemispheres. Neuroimaging techniques may show diffuse signal abnormalities involving the subcortical white matter in the parietooccipital lobes; the brain stem, basal ganglia, frontal lobes, and cerebellum may also be involved. ${ }^{1,2} \mathrm{On}$ clinical grounds, PRES is characterized by headache, seizures, obtundation and lethargy, altered mental status, and visual loss. PRES has been described in association with hypertensive encephalopathy,

Correspondence: Aurelio Negro, viale Risorgimento 80, 42100 Reggio Emilia, Italy.

Tel. +39.0522.296866/+39.0522.295896.

E-mail: negro.aurelio@asmn.re.it

Key words: preeclampsia, reversible encephalopathy, hypertension, magnetic nuclear resonance, aldosterone, plasma renin activity.

Conflict of interests: the authors declare no potential conflict of interests.

Received for publication: 3 February 2013.

Revision received: 18 March 2013.

Accepted for publication: 10 April 2013.

This work is licensed under a Creative Commons Attribution NonCommercial 3.0 License (CC BY-NC 3.0).

(C) Copyright A. Negro et al., 2013

Licensee PAGEPress, Italy

Italian Journal of Medicine 2013; 7:300-304

doi:10.4081/itjm.2013.300 eclampsia, renal failure with hypertension, or following immunosuppressive or anticancer therapy. ${ }^{1}$ Less frequently, it has been reported in the setting of autoimmune connective tissue diseases, thrombotic thrombocytopenic purpura, HIV syndrome, acute intermittent porphyria, organ transplantation, ${ }^{2}$ and hypercalcemia. $^{3}$ An abrupt rise in blood pressure is thought to be the main pathophysiological mechanism leading to an acute disruption of the bloodbrain barrier. Although being initially described as reversible, ${ }^{1}$ PRES is now believed to carry an elevated risk of permanent cerebral injury, especially if treatment is delayed. ${ }^{2,4}$ Preeclampsia (PE) is a pregnancy-related disorder, typically presenting with new-onset hypertension and proteinuria after 20 weeks of gestation, or in the early postpartum period. The term eclampsia is applied when seizures follow, complicating the preeclampsia condition. To our knowledge, a few isolated reports of PRES associated with postpartum PE have been published so far.

We report a case of PRES in the setting of severe postpartum PE associated with acquired apparent mineralocorticoid excess (AME) syndrome, possibly due to a reduced expression or activity of the placental $11 \beta$-hydroxysteroid dehydrogenase type 2 enzyme (11ß-HSD2).

\section{Case Report}

A 32-year old woman of black African origin, at her first pregnancy, was admitted at the $38^{\text {th }}$ week of gestation for scheduled caesarean delivery because of breech presentation. She had no family history of arterial hypertension. The pregnancy was uneventful. Her blood pressure at admission was $110 / 66 \mathrm{mmHg}$. 
A healthy male infant was born. She was discharged four days after delivery, with blood pressure 110/70 $\mathrm{mmHg}$. Of note, at admission, there was mild hypokalemia $(3.1 \mathrm{mEq} / \mathrm{L})$ and metabolic alkalosis $\left(\mathrm{HCO}_{3}=29 \mathrm{mEq} / \mathrm{L}\right)$. Ten days after delivery, the patient was referred to our unit because of sudden onset of thunderclap headache, with a measured blood pressure of 210/110 mmHg. Blood chemistry showed altered liver function tests (aspartate transaminase 64, alanine aminotransferase $78 \mathrm{IU} / \mathrm{L}$ ), increased lowdensity lipoprotein levels (743 IU), proteinuria (3+ at dipstick) without renal function abnormalities; serum hemoglobin, albumin, glucose and bilirubin were normal. Plasma $\mathrm{Na}^{+}$and $\mathrm{K}^{+}$concentrations were 148 and $2.9 \mathrm{mEq} / \mathrm{L}$, respectively, and persistent metabolic alkalosis $\left(\mathrm{HCO}_{3}=31 \mathrm{mEq} / \mathrm{L}\right)$ was noted; the uric acid level was $7.9 \mathrm{mg} / \mathrm{dL}$. The plasma renin activity (PRA) was clearly suppressed $(0.2 \mathrm{ngAngioI} / \mathrm{mL} / \mathrm{h})$ and the plasma aldosterone concentration (PAC) was almost undetectable $(<4 \mathrm{pg} / \mathrm{mL}$, normal range 30-140 $\mathrm{pg} / \mathrm{mL}$ ). Detailed neurological examination showed no abnormalities. Head computed tomography scan was unremarkable. We hypothesized a post partum pre-eclamptic hypertensive encephalopathy with possible appearance of PRES. A brain magnetic resonance imaging (MRI) was quickly performed and confirmed the hypothesis, showing swelling and altered signal of the brain cortex in both parietal lobes, and in the watershed zones between anterior and posterior cerebral arteries territories. A hyperintense signal was also seen, arising from the right frontal sulci, an expression of blood extravasation in the subarachnoid space, probably as a consequence of the hyperintensive crisis (Figure 1). In the regions of altered signal, diffusion-weighted imaging acquisition showed no diffusion modification. The presence of blood in the subarachnoid space prompted the acquisition of a magnetic resonance angiography study of the intracranial arterial branches (Figure 2) which excluded artero-venous malformations. A diagnosis of hypertensive encephalopathy with PRES, in the setting of post-partum PE, was made. Electroencephalography showed slow waves and diffuse irritative pattern, compatible with the above diagnosis. Renal duplex ultrasonography showed no signs of renal artery stenosis. Urinalysis showed normal excretion of fractionated metanephrine; the urinary potassium concentration was $67 \mathrm{mEq} / \mathrm{L}$, and was judged inappropriately high, in the presence of hypokalemia. The patient received intravenous labetalol for the first 24 $\mathrm{h}$ and then therapy was changed to oral amlodipine (15 mg o.d.), as blood pressure decreased to $145 / 83$ $\mathrm{mmHg}$ and her headache disappeared. After four days, blood pressure was $130 / 80 \mathrm{mmHg}$ with amlodipine $10 \mathrm{mg}$ o.d.; serum $\mathrm{K}^{+}$was $3.2 \mathrm{mEq} / \mathrm{L}$ with oral potassium supplementation. On Day 14, the patient was discharged in good clinical condition, with a normalized blood pressure, and receiving oral amlodipine $5 \mathrm{mg}$ o.d.

On MRI follow up, performed during treatment and clinical recovery, the parietal cortical lesions and the subarachnoid hemorrhage in the frontal sulci gra-

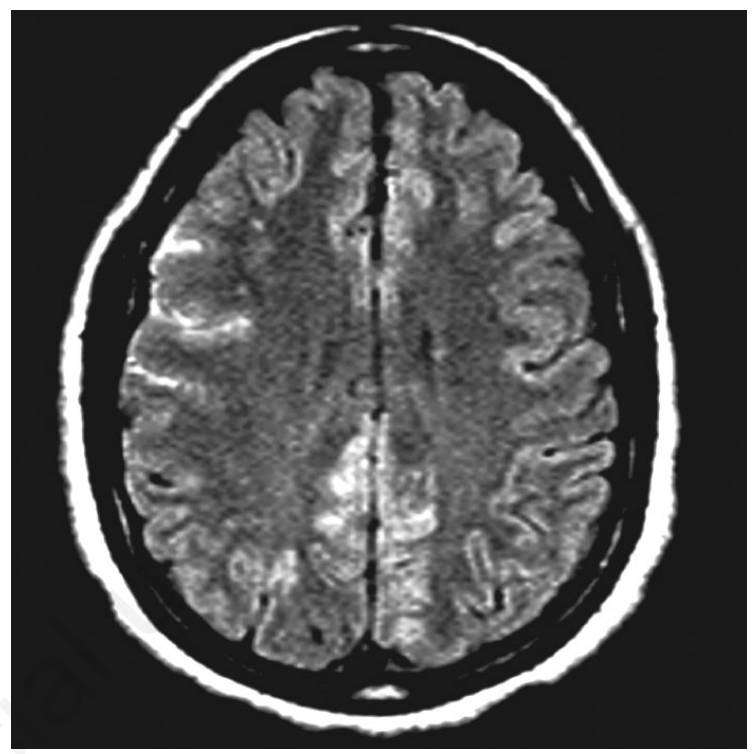

Figure 1. Magnetic resonance imaging scan (axial FLAIR-long TE; 11,000/2800/140) during acute phase showing swelling of the parietal cortex on both sides and presence of hyperintense material, presumably blood, in right frontal sulci.

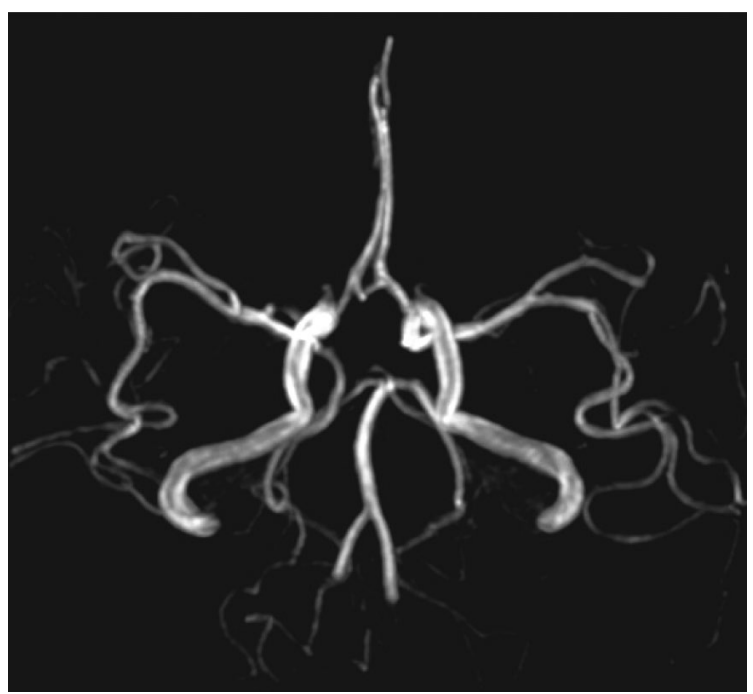

Figure 2. Magnetic resonance angiography (TOF sequence) on which no abnormality is visible. 
dually reduced and finally disappeared completely (Figure 3).

After 1.5 months, the patient was in good clinical condition, with normal blood pressure in the absence of antihypertensive therapy; she dropped oral potassium supplements after approximately three weeks. The laboratory tests showed normal serum electrolytes and no proteinuria on the dipstick. The PRA and PAC rose to $1.1 \mathrm{ngAngioI} / \mathrm{mL} / \mathrm{h}$ and $37 \mathrm{pg} / \mathrm{mL}$, respectively.

\section{Discussion}

We report a case of severe PE occurring in the late post-partum period, associated with clinical and neuroradiological findings consistent with PRES. PE is a multi-organ disease, characterized by hypertension and proteinuria, possibly complicated by eclamptic seizures. It affects approximately $5 \%$ of all pregnancies worldwide, bearing significant morbidity and mortality for the mother and the fetus. The disease typically occurs late in pregnancy, during the third trimester or in the early post-partum period. Although the etiology of PE is unclear, there is compelling evidence pointing to a widespread vascular endothelial cell dysregulation and dysfunction as a result of placental hypoperfusion, resulting in placental secretion of antiangiogenic growth factors. ${ }^{5}$ However, a significantly reduced concentration and activity of the enzyme $11 \beta-H S D 2$ has been described in human placenta of patients with PE. ${ }^{6}$ This enzyme normally converts serum cortisol to its inactive metabolite

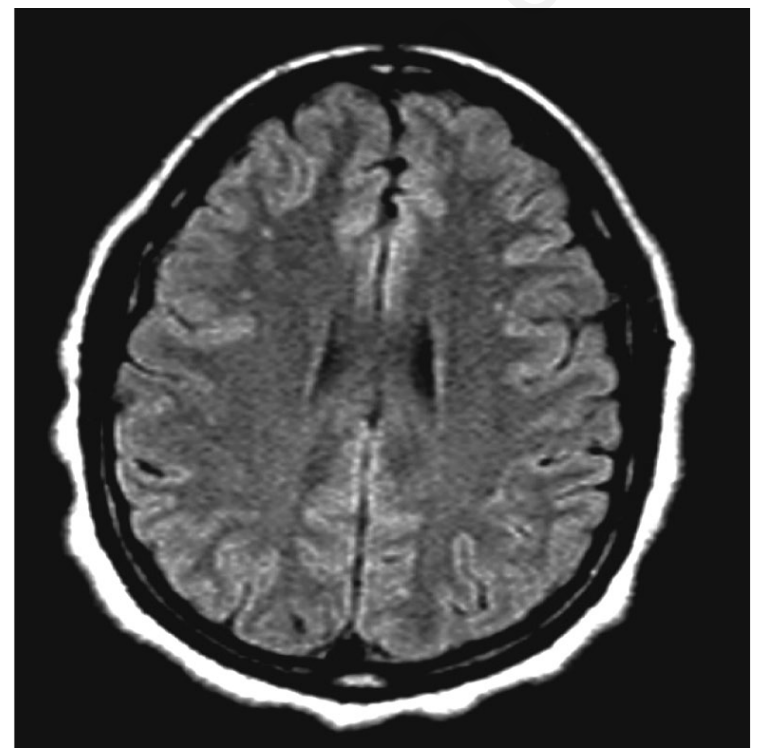

Figure 3. One month after the first imaging study, and after treatment with good clinical results, the magnetic resonance imaging FLAIR appears completely normalized. cortisone. The $11 \beta-\mathrm{HSD} 2$ is predominantly expressed, along with the mineralocorticoid receptor, in the renal distal tubules and collecting ducts, in the colon, in the salivary glands and also in the placenta, where it protects the fetus from an excessive amount of maternal cortisol. ${ }^{7}$ In fact, the enzyme regulates access of glucocorticoid hormones to glucocorticoid and mineralocorticoid receptors. Since cortisol, but not cortisone, is a potent agonist of mineralocorticoid receptors and circulates at levels 100 to 1000 times higher than those of aldosterone, a reduced activity of the $11 \beta-\mathrm{HSD} 2$ may expose the kidney to an excess of cortisol, which can act as a potent mineralocorticoid hormone with ensuing salt retention, hypertension, hypokalemia and metabolic alkalosis. ${ }^{8}$ The AME syndrome is the result of defective $11 \beta$-HSD2 enzyme. It is characterized clinically by a mineralcorticoid excess status, with hypertension, hypokalemia and metabolic alkalosis, plasma volume expansion and a marked suppression of the renin-angiotensin-aldosterone system. First described by Ulick and colleagues, ${ }^{9}$ the AME syndrome can be congenital or acquired, but the two forms share the same pathophysiology. Congenital AME syndrome has an autosomal recessive model of inheritance. AME patients are homozygous for $11 \beta$-HSD2 inactivating mutations causing full or partial loss of enzymatic activity; the onset of symptoms may occur in childhood, adolescence or early adulthood, with phenotypes varying from life-threatening conditions to milder forms. In most cases, the acquired AME syndrome is due, for instance, to excessive liquorice intake. Biochemical abnormalities comprise suppressed PRA, undetectable serum aldosterone levels, hypokalemia, metabolic alkalosis, altered ratio of urinary free cortisol (UFF) to free cortisone (UFE).$^{10}$ The urinary $\mathrm{UFF} / \mathrm{UFE}$ ratio is not widely used, and, in our case, it was unfortunately not available.

In our patient, we suspected an acquired AME syndrome due to a reduced 11 $\beta$-HSD2 activity of placental origin on the basis of hypokalemia with alkalosis and renal potassium wasting, marked suppression of PRA and serum aldosterone levels and subsequent progressive recovery of the renin-aldosterone system. The definite diagnosis of AME syndrome was not fully established due to the absence of urinary UFF/UFE ratio. However, we acknowledge that it is hard to believe that a putative impairment in placental $11 \beta-H S D 2$ activity per se may have been involved in the pathogenesis of a systemic syndrome encompassing accelerated hypertension and deranged autoregulation of the cerebral circulation. PRA and plasma aldosterone are reduced in PE compared to normal pregnancy, together with an increase in the sensitivity of the vessels to vasoconstrictor agents such as angiotensin II (AII). However, plasma aldosterone levels are usually less suppressed than those of PRA, so that the so-called al- 
dosterone-to-renin ratio is approximately 2 -fold greater in PE than in normal pregnancy. This does not appear to be due to changes in potassium, atrial natriuretic peptide, dopamine or adrenocorticotropic hormone, and may be another manifestation of increased sensitivity to AII in PE. ${ }^{11}$

Another putative explanation for the clinical and laboratory observations in our patient could be the presence of circulating agonistic AT1-receptor autoantibodies (AT1-RAA) that have been implicated in the pathogenesis of PE. In vitro and in vivo studies have determined the role of this AT1-RAA in triggering PE. It has been observed that in human trophoblast cells AT1-RAA substances induce the generation of reactive oxygen species intracellularly through nicotinamide adenine dinucleotide phosphate-oxidase activation. In addition, these same cells stimulate the release of PAI-1, resulting in a decreased trophoblast invasiveness, generating defective placentation. This increase in PAI-1 is also observed in mesangial cells, which can produce a decrease in extracellular matrix degradation and increased subendothelial fibrin deposition, thereby determining renal damage leading to proteinuria and a decreased glomerular filtration rate. It has also been observed that AT1-RAA binds to endothelial and vascular cells, causing endothelial damage and vasoconstriction. In animal models, it has been observed that the inoculation of AT1-RAA from patients with PE is capable of reproducing the characteristics of the disease. Reports in rats showed that surgically induced placental ischemia may cause increased levels of AT1-RAA and trigger hypertension and proteinuria. In the same way, pregnant human studies show that placental perfusion abnormality is associated with increased plasma concentrations of AT1-RAA before the onset of the disease, and the plasmatic levels of these autoantibodies correlate with the severity. ${ }^{12}$

The elevated levels of AT1-RAA may explain the hypersensitivity to the effects of AII. However, increases in AT1-RAA are not specifically related to PE, as this autoantibody is also elevated in intrauterine growth restriction without PE. Therefore, the role of AT1-RAA as mediators of pre-eclamptic phenotype is doubtful. This is corroborated by the fact that the disappearance of clinical symptoms does not require the loss of autoantibodies..$^{13}$

Posterior reversible encephalopathy syndrome is an uncommon diagnosis in day-to-day obstetric care, but it is known to be part of pregnancy-induced hypertension and eclampsia. It has been recently reviewed by Verma et al. ${ }^{14}$ The clinical presentation of PRES includes headache, altered mental status, visual disturbances including cortical blindness, seizures, hypertension, and focal or lateralizing neurological signs. Although the pathogenesis of PRES remains poorly understood, the most widely accepted mechanism is that a sudden rise in blood pressure may exceed the upper limit of cerebral autoregulation, with abrupt dilation of cerebral arterioles resulting in interstitial extravasation of serum protein and fluid, i.e. vasogenic edema. In the rarer normotensive cases occurring after immunosuppressive or cytotoxic treatment, a toxic effect on vascular endothelium is hypothesized. ${ }^{2}$ MRI plays an important role in providing early information about cerebral involvement by diffuse signal abnormalities mainly involving the subcortical white matter in the parieto-occipital regions of the brain; the temporal and frontal lobes, cerebellum, basal ganglia, and brainstem may also be involved. ${ }^{1,2}$ Although most cases of PRES are reversible, delay in diagnosis and treatment may lead to irreversible lesions. ${ }^{2}$ Therefore, early recognition and correction of the condition underlying PRES is the recommended treatment for this disorder. The appropriate and rapid lowering of blood pressure in patients with PRES is essential to avoid permanent brain injury.

\section{Conclusions}

This case illustrates a late postpartum severe PE complicated by PRES associated with suppressed plasma aldosterone levels and PRA. The presence of even mild and isolated derangements in serum potassium and acid-base balance, in the setting of PE, should prompt clinicians to broaden the spectrum of differential diagnoses, including acquired AME syndrome.

\section{References}

1. Hinchey J. A reversible posterior leukoencephalopathy syndrome. N Engl J Med 1996;334:494-500.

2. Garg RK. Posterior leukoencephalopathy syndrome. Postgrad Med J 2001;77:24-8.

3. Kastrup O, Maschke M, Wanke I, et al. Posterior reversible encephalopathy syndrome due to severe hypercalcemia, J Neurol 2002;249:1563-6.

4. Ay H, Buonanno FS, Schaefer PW, et al. Posterior leukoencephalopathy without severe hypertension: Utility of diffusion-weighted MRI. Neurology 1998;51: $1369-76$.

5. Jankowski M. Angiogenesis inhibition and preeclampsia. J Hypertens 2009;27:707-8.

6. Schoof E, Girstl M, Frobenius W, et al. Decreased gene expression of 11 $\beta$-hydroxysteroid dehydrogenase type 2 and 15-Hydroxyprostaglandin dehydrogenase in human placenta of patients with preeclampsia. J Clin Endocrinol Metab 2001;86:1313-7.

7. Yang K. Placental 11 $\beta$-hydroxysteroid dehydrogenase: barrier to maternal glucocorticoids. Rev Reprod 1997;2: 129-32.

8. Stewart PM. Mineralocorticoid hypertension. Lancet 1999;353:1341-7.

9. Ulick S, Levine LS, Gunczler P, et al. A syndrome of apparent mineralocorticoid excess associated with defects 
in the peripheral metabolism of cortisol. J Clin Endocrinol Metab 1979;49:757-64.

10. Negro A, Rossi E, Regolisti G, et al. Liquorice-induced sodium retention. Merely an acquired condition of apparent mineralocorticoid excess? A case report. Ann Ital Med Int 2000;15:296-300.

11. Brown MA, Wang J, Whitworth JA. The renin-angiotensin-aldosterone system in pre-eclampsia. Clin Exp Hypertens 1997;19:713-26.
12. Rodriguez M, Moreno J, Hasbun J. RAS in pregnancy and preeclampsia and eclampsia. Int J Hypertens 2012; 2012:739274.

13. Verma A, Shaji PD. A patient with postpartum hypertension and seizure. Am J Kidney Dis 2011;58:A29-31.

14. Liao YH, Wei YM, Wang M, et al. Autoantibodies against AT1-receptor and $\alpha 1$-adrenergic receptor in patients with hypertension. Hypertension Res 2002; 25:641-6. 